\title{
Podoplanin is involved in the prognosis of head and neck squamous cell carcinoma through interaction with VEGF-C
}

\author{
HYUN-YI KIM ${ }^{1}$, KI-SANG RHA ${ }^{1}$, GEUN AE SHIM ${ }^{1}$, JU-HEE KIM ${ }^{1}$, JIN-MAN KIM ${ }^{2}$, \\ SONG MEI HUANG ${ }^{2}$ and BON SEOK KOO ${ }^{1}$

\begin{abstract}
${ }^{1}$ Department of Otolaryngology - Head and Neck Surgery, Cancer Research Institute, Research Institute for Medical Sciences and ${ }^{2}$ Department of Pathology, Chungnam National University College of Medicine, Daejeon 301-721, Republic of Korea
\end{abstract}

Received March 20, 2015; Accepted June 2, 2015

DOI: $10.3892 /$ or.2015.4070

\begin{abstract}
We investigated the clinical significance of podoplanin expression in relation to clinicopathological variables in head and neck squamous cell carcinoma (HNSCC), to determine its effectiveness as a marker for high-risk HNSCC patients. Upregulation of podoplanin in HNSCC tissues was examined using immunohistochemistry and clinicopathological analyses. Wound healing and invasion assays were performed using HNSCC cells that were transfected with podoplanin siRNA, podoplanin vector and cotransfection with both the podoplanin vector and VEGF-C siRNA. High podoplanin expression was significantly associated with $\sim 3$ and 5-fold increases in the presence of positive lymph node metastasis and poor histological grade, respectively $(\mathrm{P}<0.05)$. High levels of podoplanin expression were significantly associated with decreased overall and disease-specific survival rates $(\mathrm{P}<0.05)$. Furthermore, upregulation of podoplanin induced cell wound repair activity and invasiveness in the FaDu and SNU-1041 cells, respectively, while downregulation of podoplanin expression through VEGF-C silencing using co-transfection of both the podoplanin vector and VEGF-C siRNA suppressed cell wound healing and invasion abilities in the HNSCC cells. Our findings suggest that high podoplanin expression is associated with aggressive tumor behavior, poor prognosis and metastatic regulation through interaction with VEGF-C, suggesting that podoplanin may be used as a potential prognostic biomarker for HNSCC.
\end{abstract}

Correspondence to: Professor Bon Seok Koo, Department of Otolaryngology - Head and Neck Surgery, Cancer Research Institute, Research Institute for Medical Sciences, Chungnam National University College of Medicine, 640 Daesa-Dong, Chung-Gu, Daejeon 301-721, Republic of Korea

E-mail: bskoo515@cnuh.co.kr

Key words: head and neck squamous cell carcinoma, podoplanin, lymph node metastasis, invasion, prognosis

\section{Introduction}

Head and neck squamous cell carcinoma (HNSCC) is the sixth most common type of cancer worldwide (1). HNSCC patients have benefited greatly from recent advances in surgical techniques, radiotherapy and chemotherapy. However, despite the advances in local control and overall quality of life achieved with the use of combined therapies, the survival rate for HNSCC has not improved significantly over the last two decades (2). Invasion of normal adjacent tissue and lymph node metastasis are the most adverse independent prognostic factors for patients with $\operatorname{HNSCC}(3,4)$.

Although the current TNM staging system is used routinely, its value for predicting clinical outcomes remains modest, particularly among tumors of identical TNM stage $(5,6)$. Thus, there is a continuing need to identify biological markers that may be able to augment the clinical staging system. It is also believed that underlying molecular features of tumors play an essential role in determining their aggressiveness.

Podoplanin is a mucin-type transmembrane glycoprotein that is specifically expressed in lymphatic, but not in blood endothelial cells (7). It has been shown in a knockout animal model that podoplanin deficiency causes lymphovascular malformations associated with congenital lymphedema and dilation of lymphatic vessels, suggesting that under physiological conditions, podoplanin may play an important role in regulating peripheral lung cell proliferation and lymphatic vascular development (8). Recent studies have reported podoplanin expression in carcinomas of the skin, lung, uterus, esophagus and squamous cell carcinomas of the oral cavity (9-15). Moreover, high expression of podoplanin was found to be significantly associated with poor prognosis, suggesting that podoplanin may have biological functions in tumor cells $(9,12-15)$.

In the present study, we investigated the prognostic influence of podoplanin expression in HNSCC and its association with clinicopathological variables, particularly regional lymph node metastasis, to determine its effectiveness as a marker of high-risk HNSCC. To verify the involvement of podoplanin in the metastasis of HNSCC, we examined cell wound healing migration and invasion assays using both RNA interference and overexpression of podoplanin. 


\section{Patients and methods}

Patients. This retrospective study included 119 consecutive treatment-naïve patients with biopsy confirmed primary HNSCC, who were treated at the Department of Otolaryngology, Head and Neck Surgery, Chungnam National University Hospital between 1998 and 2010. All patients underwent primary surgery including neck dissection. Tumor staging was conducted according to the criteria of the $7^{\text {th }}$ edition of the AJCC (16). Clinicopathological parameters, including histopathological and surgical studies, were obtained from the medical charts.

Follow-up data were collected by a combination of chart review and the local government office for the registration of residents. At the time of the analysis, 51 (42.9\%) patients had succumbed to the disease; 45 (37.8\%) of them due to the tumor. Overall, 54 patients developed recurrent disease, including 21 (17.6\%) local recurrences, 29 (24.3\%) subsequent regional lymph node metastases and 8 (6.7\%) distant recurrences. The average follow-up time was 40.6 (range, 2-144) months.

Tissue processing and immunohistochemical analysis. The immunohistochemical (IHC) study of the HNSCC patients was approved by the local institutional review board. Formalin-fixed and paraffin-wax-embedded tissues from 119 HNSCC lesions - 44 in the oral cavity, 17 in the oropharynx, 48 in the larynx and 10 in the hypopharynx - were retrieved from the archives of the Department of Pathology, Chungnam National University Hospital, Korea. Sections ( $4 \mu \mathrm{m})$ of the paraffin-wax-embedded tissue blocks were used for IHC studies according to the procedures provided in the Vectastain ABC kit (Vector, Burlingame. CA, USA). All slides were scored, as described by Yuan et al (14), by at least two pathologists who were blinded to the clinical information of the patients. For scoring, representative areas of each tissue section were selected and evaluated independently. Cytoplasm and/or membrane immunoreactivity was considered to indicate podoplanin expression. Quantitative scores of 0 to 5 were assigned when $0,1-10,11-30,31-50,51-80$ or $81-100 \%$ of the tumor cells were positive, respectively. The staining intensity was rated on a scale of 0 to 3 : $0=$ negative, $1=$ weak, $2=$ moderate, and $3=$ strong. The raw data were then converted to a German Immunoreactive Score (IRS) by multiplying the quantity and staining intensity scores. Theoretically, the scores may range from 0 to 15 . An IRS score above the median $(\geq 7)$ was considered high reactivity and $0-6$, low. Consensus opinions were used to assign final IRS scores in disputed cases before data analysis.

Cell lines and culture conditions. The HNSCC cell lines FaDu and SNU-1041 were purchased from the American Type Culture Collection (ATCC; Rockville, MD, USA) and the Korean Cell Line Bank (KCLB; Seoul, Korea) and maintained in DMEM and RPMI supplemented with $100 \mathrm{U} / \mathrm{ml}$ penicillin, $100 \mu \mathrm{g} / \mathrm{ml}$ streptomycin, $25 \mathrm{ng} / \mathrm{ml}$ amphotericin B and $10 \%$ fetal bovine serum (FBS) (Gibco, Carlsbad, CA, USA) at $37^{\circ} \mathrm{C}$ in a humidified incubator with $5 \% \mathrm{CO}_{2}$, respectively.

siRNA knockdown of podoplanin gene expression. Podoplanin-specific siRNA and control siRNA were purchased from Bioneer (Daejeon, Korea). The targeted sequences of podoplanin were sense siRNA, 5'-GAU AAC ACG UGU GGU GAA CAA TT-3' and antisense, 5'-UUG UUC ACC ACG UGU UAU GTT-3'. Podoplanin siRNA transfection was performed in opti-MEM media with the transfection reagent Lipofectamine 2000 (Invitrogen Life Technologies, Carlsbad, CA, USA) following the manufacturer's instructions.

Gene manipulation of podoplanin. Human podoplanin cDNA (NM_006474.4) was PCR-amplified from a cDNA library which was purchased from Origene (Rockville, MD, USA) and cloned into the NotI/NotI site of pCMV6-XL5 (Origene). The PDPN vector containing the podoplanin coding region or the mock vector was transfected into the FaDu or SNU-1041 cell line with Lipofectamine 2000 according to the manufacturer's instructions, respectively.

$R T-P C R$. Oligonucleotides of the VEGF family genes such as VEGF-A, -B, -C and -D were purchased from Bioneer. The primer sequences for GAPDH were 5'-CCATCACCATCT TCCAGGAG-3' (sense) and 5'-ACAGTCTTCTGGGTGGCA GT-3' (antisense). The VEGF family-specific primers used for PCR were described in a previous study (17). Relative gene expression levels were normalized to GAPDH expression.

Western blot analysis. Sodium dodecyl sulfate-polyacrylamide gel electrophoresis (SDS-PAGE) was conducted using a Mini-Protean Tetra Cell (Bio-Rad, Hercules, CA, USA) and a $12 \%$ gel according to the manufacturer's instructions. Proteins were transferred to a PVDF membrane and probed with primary antibodies followed by an HRP-conjugated secondary antibody. Immunolabeled proteins were detected by incubation with enhanced chemiluminescence (ECL) substrate followed by exposure of the membrane to autoradiography film.

Wound healing cell migration assays. Cells were plated on a 60-mm culture dish with $90 \%$ confluence and an injury line with a width of $2 \mathrm{~mm}$ was made by scraping across the cell monolayer with a yellow tip. After floating cell debris was removed by washing with PBS, cell migration was monitored under a phase-contrast microscope and photographed.

Cell invasion assays. FaDu and SNU-1041 cells were cultured for $24 \mathrm{~h}$ after the transfection of podoplanin siRNA or PDPN vector in growth medium containing $10 \%$ FBS. The following procedures are described in a previous study (18). The cells were counted by taking photomicrographs at a magnification, x100. Cells in five different fields of each well were counted with two wells per treatment. The mean values were obtained from three replicate experiments and were subjected to a t-test.

Statistical analysis. The SPSS software (ver. 14; SPSS, Chicago, IL, USA) was used to perform statistical analyses. Univariate analysis of podoplanin expression and clinicopathological parameters was performed using the Fisher's exact test. Significant variables in the univariate analysis were included in a multinomial logistic regression test (multivariate analysis). The Kaplan-Meier method (assessed by log-rank test) and Cox regression model were used for univariate and multivariate overall and disease-specific survival analyses. A P-value $<0.05$ was considered to indicate statistical significance. 

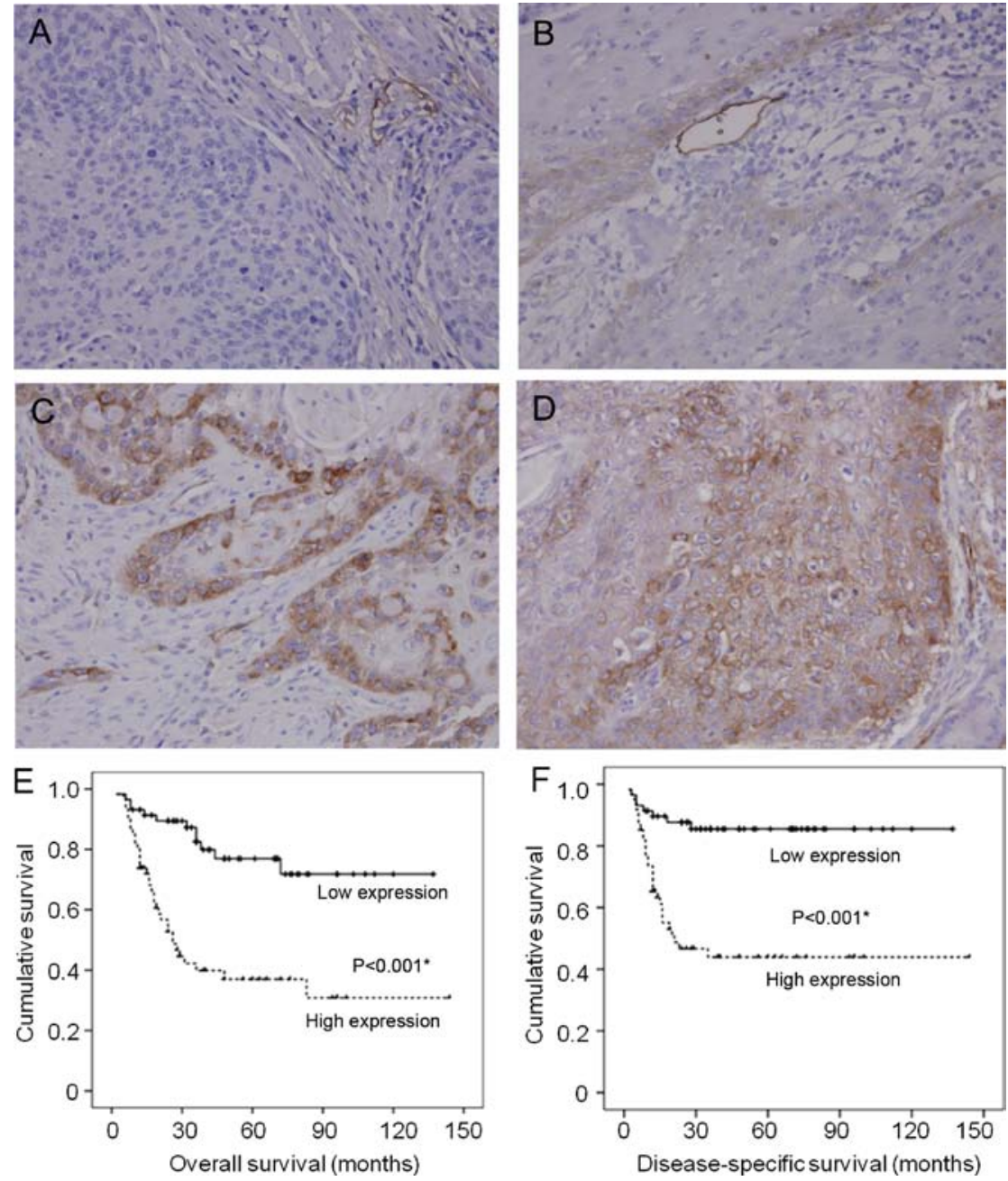

Figure 1. Representative micrographs showing podoplanin staining intensity and the overall survival rate of HNSCC patients. (A) Tumor cells without podoplanin expression at magnification, $\mathrm{x} 400$ (score 0). (B) Tumor with weak podoplanin expression at magnification, $\mathrm{x} 400$ (score 1); (C) moderate expression of podoplanin at magnification, $\mathrm{x} 400$ (score 2); (D) strong podoplanin expression at magnification, $\mathrm{x} 400$ (score 3). (E) Overall survival rate of patients whose tumors expressed high and low levels of podoplanin. (F) Disease-specific survival rate of patients whose tumors expressed high and low levels of podoplanin. HNSCC, head and neck squamous cell carcinoma.
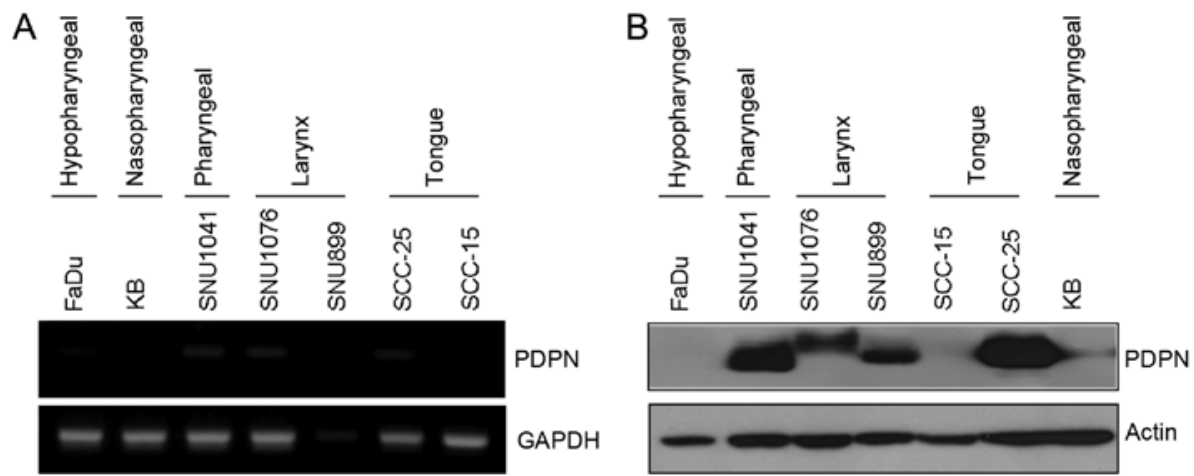

Figure 2. Expression of podoplanin in HNSCC cell lines using RT-PCR (A) and western blot analysis (B). The GAPDH or actin gene was used as an internal reaction control, respectively. HNSCC, head and neck squamous cell carcinoma.

\section{Results}

Upregulation of podoplanin expression in HNSCC tissues and cell lines. As expected, podoplanin was highly expressed in the endothelial cells of lymphatic vessels, but was not detectable in the endothelial cells of blood vessels. In histologically normal squamous epithelium adjacent to the tumors, podoplanin expression was either not detectable or extremely low in some basal cells. In primary HNSCC, podoplanin expression was generally heterogeneous and differentially increased (Fig. 1). Podoplanin transcript and translational levels were expressed differentially in the HNSCC cell lines (Fig. 2). 
Table I. Associations between podoplanin expression and the clinicopathological features of the patients with HNSCC.

\begin{tabular}{|c|c|c|c|c|}
\hline \multirow[b]{2}{*}{ Variables } & \multirow{2}{*}{$\begin{array}{l}\text { No. of } \\
\text { patients }\end{array}$} & \multicolumn{2}{|c|}{$\begin{array}{l}\text { Podoplanin } \\
\text { expression }\end{array}$} & \multirow[b]{2}{*}{ P-value } \\
\hline & & Low & High & \\
\hline \multicolumn{5}{|l|}{ Age (years) } \\
\hline$<65$ & 63 & 28 & 35 & \multirow[t]{2}{*}{0.361} \\
\hline$\geq 65$ & 56 & 30 & 26 & \\
\hline \multicolumn{5}{|l|}{ Gender } \\
\hline Male & 105 & 49 & 56 & \multirow[t]{2}{*}{0.262} \\
\hline Female & 14 & 9 & 5 & \\
\hline \multicolumn{5}{|l|}{ T stage } \\
\hline I+II & 66 & 36 & 30 & \multirow[t]{2}{*}{0.197} \\
\hline III+IV & 53 & 22 & 31 & \\
\hline \multicolumn{5}{|c|}{ Lymph node metastasis } \\
\hline No & 60 & 38 & 22 & \multirow[t]{2}{*}{$0.002^{\mathrm{a}}$} \\
\hline Yes & 59 & 20 & 39 & \\
\hline \multicolumn{5}{|l|}{ AJCC stage } \\
\hline $\mathrm{I}+\mathrm{II}$ & 41 & 27 & 14 & \multirow[t]{2}{*}{$0.008^{\mathrm{a}}$} \\
\hline III+IV & 78 & 31 & 47 & \\
\hline \multicolumn{5}{|c|}{ Histological grade } \\
\hline Well & 33 & 23 & 10 & \multirow[t]{3}{*}{$0.007^{\mathrm{a}}$} \\
\hline Moderate & 56 & 26 & 30 & \\
\hline Poor & 30 & 19 & 21 & \\
\hline Primary site & & & & 0.465 \\
\hline Oral cavity & 44 & 24 & 20 & \\
\hline Oropharynx & 17 & 10 & 7 & \\
\hline Hypopharynx & 10 & 4 & 6 & \\
\hline Larynx & 48 & 20 & 28 & \\
\hline
\end{tabular}

${ }^{\mathrm{a}} \mathrm{P}<0.05$ between the two categories for a given variable. HNSCC, head and neck squamous cell carcinoma.

Relationships of podoplanin expression with clinicopathological features in patients with HNSCC. Immunohistochemical staining images are shown in Fig. 1. Of the 119 cases, $26(21.8 \%)$ showed no podoplanin expression, 32 (26.9\%) had weak, 37 (31.1\%) moderate and 24 (20.2\%) strong expression. For statistical purposes, we divided the cases into two groups; those with scores (IRS) $\leq 6$ (the median value) were considered to have low podoplanin expression, whereas those with scores $>6$ were considered to have high expression. Thus, podoplanin expression was low in 58 (48.7\%) cases and high in 61 (51.3\%).

Relationships between the degree of podoplanin expression and the clinicopathological features of the 119 HNSCC patients are shown in Table I. There was a statistically significant correlation between high podoplanin expression and the presence of lymph node metastasis, advanced AJCC stage, and poor histological grade. In the multivariate analysis, high podoplanin expression was significantly associated with $\sim 3$ - and 5-fold increases in the presence of positive lymph node metastasis and poor histological grade, respectively $(\mathrm{P}<0.05$; Table II).

Correlation of podoplanin expression and the overall survival and disease-specific survival rates of the HNSCC patients. High podoplanin expression had a marked impact on the overall $(\mathrm{P}<0.001)$ and disease-specific survival rate $(\mathrm{P}<0.001$; Fig. 1E and F). The 5-year overall and disease-specific survival rates were 77 and $86 \%$, respectively, in patients with low podoplanin expression, while 37 and $44 \%$, respectively, in those with high podoplanin expression.

Cox proportional hazards regression analysis was performed to determine whether the effect of podoplanin expression on the disease-specific survival rate was dependent on other known risk factors. Poor histological grade and high podoplanin expression were independent significant factors for worse disease-specific survival rates $(\mathrm{P}<0.05$; Table III). The disease-specific death risk in HNSCC patients with high podoplanin expression was $\sim 3$-fold higher than in those with a lower podoplanin expression (Table III).

Silencing of podoplanin suppresses wound healing cell migration and invasion in HNSCC. After cells were transfected with the podoplanin siRNA for $24 \mathrm{~h}$, total protein was extracted, and western blot analysis was performed. There were various degrees of podoplanin protein suppression, depending on the cell types and there was no change in the cellular morphology of cells that were treated with podoplanin siRNA (data not shown). As shown in Fig. 3A, control siRNA-expressing FaDu or SNU-1041 cells repaired the wounded area rapidly at $24 \mathrm{~h}$. Meanwhile, treatment with podoplanin siRNA in FaDu and SNU-1041 cells inhibited wound repair significantly at $24 \mathrm{~h}$, respectively. In the Transwell invasion assay, the percentages of the invasive cells in the podoplanin siRNA-expressing FaDu and SNU-1041 were decreased to 72 and $80.7 \%$ of that

Table II. Multinomial logistic regression for the associations of podoplanin expression with lymph node metastasis, AJCC stage and histological grade.

\begin{tabular}{lcccc}
\hline Factor & $\beta$ & P-value & $\operatorname{Exp}(\beta)$ & $95 \%$ CI \\
\hline Positive lymph node metastasis & 1.153 & $0.031^{\mathrm{a}}$ & 3.168 & $(1.108-9.059)$ \\
Advanced AJCC stage & 0.005 & 0.993 & 1.005 & $(0.326-3.098)$ \\
Poor histological grade & 1.604 & $0.006^{\mathrm{a}}$ & 4.972 & $(1.581-15.630)$ \\
\hline
\end{tabular}

$\operatorname{Exp}(\beta)$ indicates odds ratio; $\mathrm{CI}$, confidence interval. ${ }^{\mathrm{a}}<0.05$ between the two categories for a given variable. 
Table III. Multivariate Cox regression analysis of disease-specific death events in the HNSCC patients $(n=119)$.

\begin{tabular}{lccc}
\hline Parameters & Risk ratio (RR) & $95 \%$ CI & P-value \\
\hline Age (years) & 1.195 & $0.598-2.388$ & 0.614 \\
Gender & 0.421 & $0.122-1.449$ & 0.170 \\
Advanced T stage & 0.607 & $0.284-1.293$ & 0.196 \\
Lymph node metastasis & 2.293 & $0.952-5.520$ & 0.064 \\
Advanced AJCC stage & 4.438 & $0.993-19.831$ & 0.051 \\
Poor histological grade & 17.027 & $2.220-130.574$ & $0.006^{\mathrm{a}}$ \\
High podoplanin expression & 2.981 & $1.320-6.732$ & $0.009^{\mathrm{a}}$
\end{tabular}

${ }^{\text {a}} \mathrm{P}<0.05$ between the two categories for a given variable. HNSCC, head and neck squamous cell carcinoma. CI, confidence interval.

A
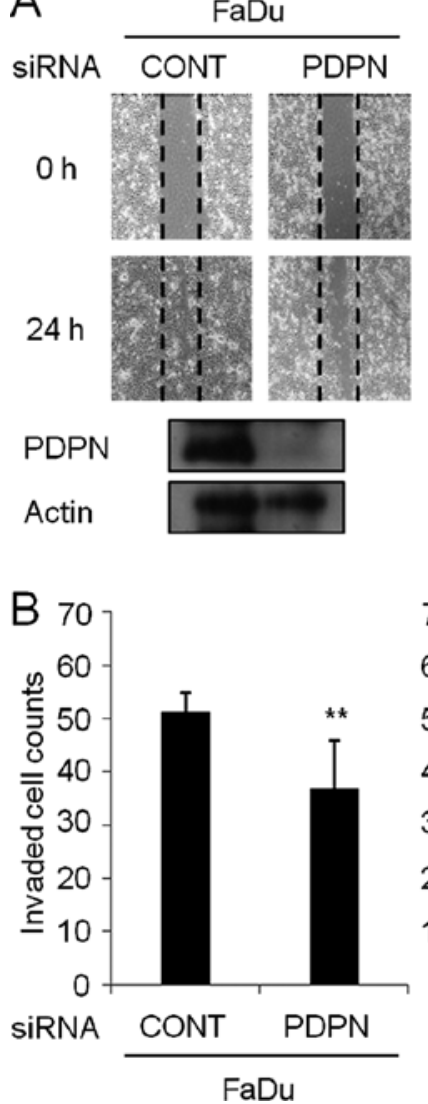
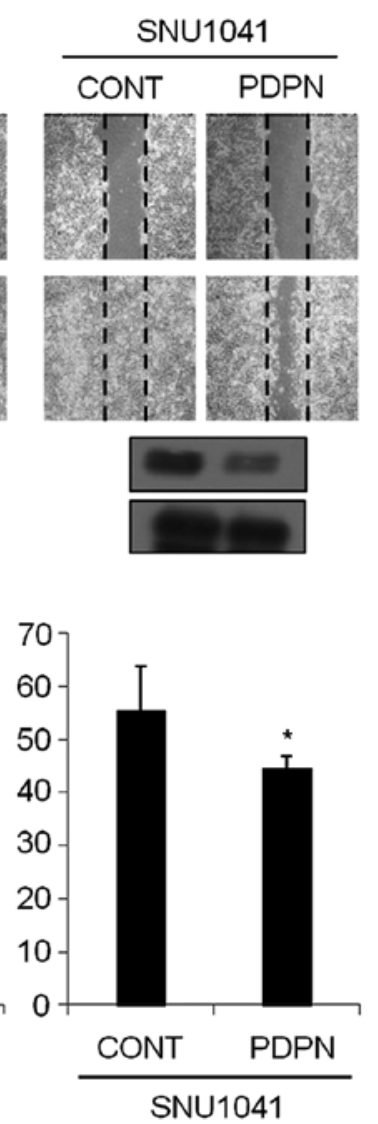

C

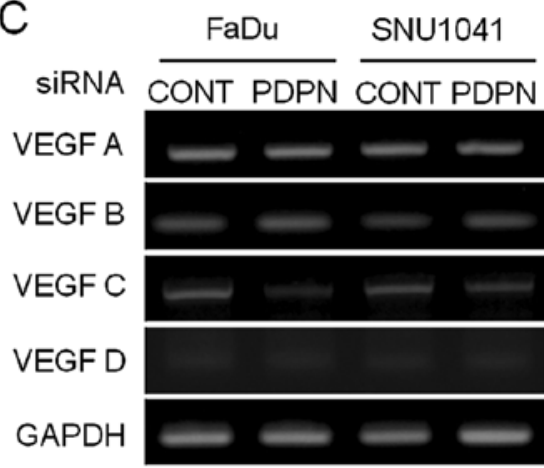

D

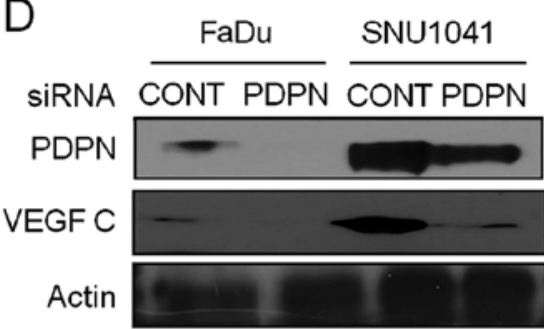

Figure 3. Inhibition of cell wound healing migration and invasion in podoplanin siRNA-expressing HNSCC cells. (A) The wound healing assay was conducted by scraping a wound area in $90 \%$ confluent FaDu and SNU-1041 cells, respectively. Cells were incubated after transfection with 100 pmol podoplanin siRNA and were monitored using phase-contrast microscopy after $24 \mathrm{~h}$. (B) The invasion assay was carried out according to the invasion assay method described in Material and methods. Invaded cells were counted by capturing photomicrographs at magnification, x100. Representative fields were photographed. Invaded cells in each well were counted in five different fields under the microscope. The mean values and the standard error were obtained from three individual experiments. $\mathrm{P}<0.1$ and ${ }^{* *} \mathrm{P}<0.05$. (C) RT-PCR analyses of VEGF-A, -B, -C and -D were performed in podoplanin siRNA-expressing FaDu and SNU-1041 cells compared to control siRNA-expressing cells, respectively. (D) Western blot analysis of expression of podoplanin and VEGF-C in podoplanin siRNAexpressing FaDu and SNU-1041 cells, respectively. Actin expression was used as an internal control.

in the control siRNA-expressing FaDu and SNU-1041 cells, respectively (Fig. 3B). In addition, inhibition of podoplanin led to suppression of the VEGF-C transcript level (Fig. 3C) and protein expression (Fig. 3D) in the FaDu and SNU-1041 cells, respectively. Other VEGF family such as VEGF-A, -B and -D were not altered in the podoplanin siRNA-expressing cells when compared to levels in the control cells (Fig. 3C).
Overexpression of podoplanin induces wound healing cell migration and invasion in the HNSCC cells. As shown in Fig. 4A, mock vector-expressing FaDu or SNU-1041 cells slightly repaired the wounded area at $24 \mathrm{~h}$. However, PDPN vector-overexpressing $\mathrm{FaDu}$ and SNU-1041 cells induced wound repair rapidly at $24 \mathrm{~h}$, respectively. In a Transwell invasion assay, the percentages of invasive cells in the 
A

Oh

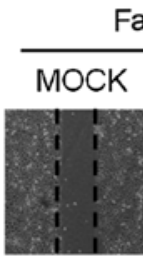

$24 \mathrm{~h}$
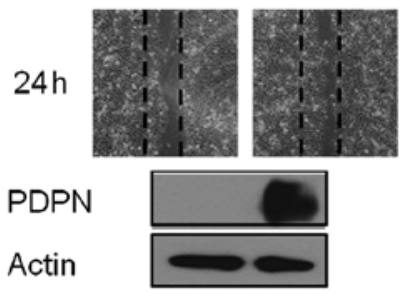

B

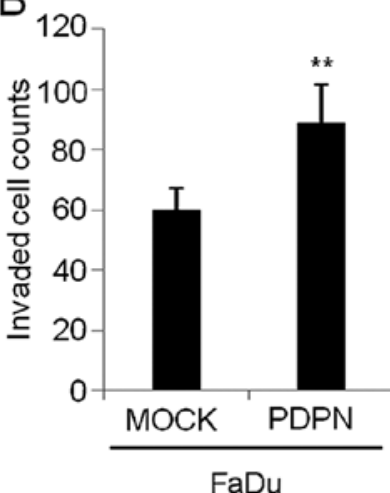

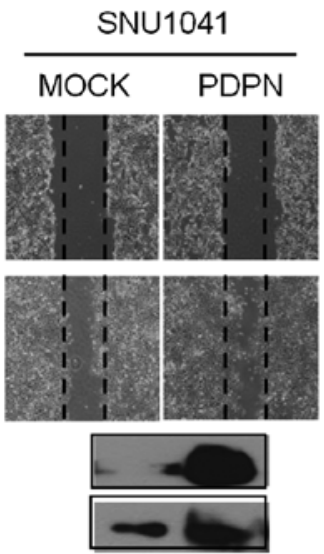

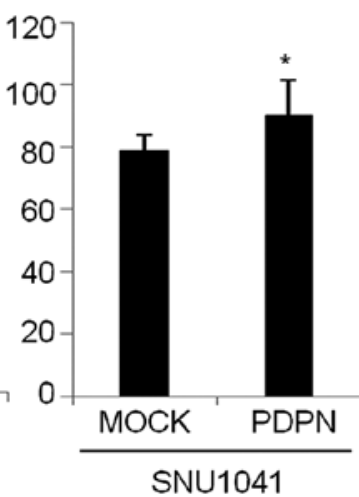

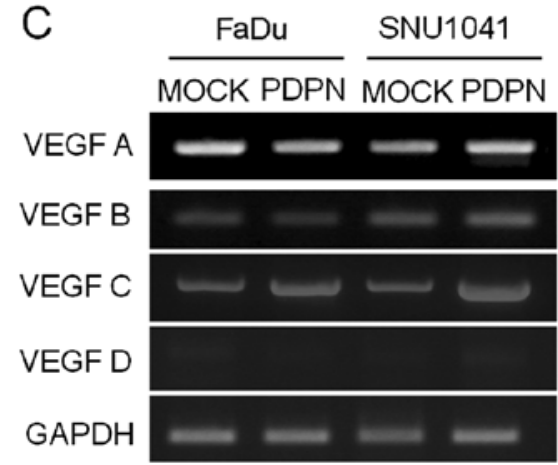

D

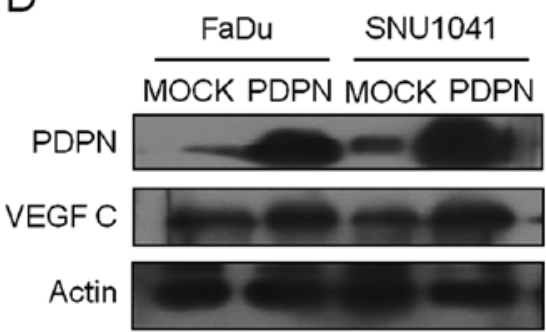

Figure 4. Induction of cell wound healing migration and invasion in the podoplanin-overexpressing FaDu and SNU-1041 cells. (A) The wound healing assay was conducted by scraping a wound area. Cells were monitored using phase-contrast microscopy at $24 \mathrm{~h}$ after transfection of podoplanin vector. (B) The invasion assay was carried out according to the invasion assay method described in Patients and methods. The mean values and the standard error were obtained from three individual experiments. ${ }^{*} \mathrm{P}<0.1$ and ${ }^{* *} \mathrm{P}<0.05$. (C) RT-PCR analyses of VEGF-A, $-\mathrm{B},-\mathrm{C}$ and $-\mathrm{D}$ were performed in podoplanin-overexpressing cells compared to mock cells. (D) Western blot analysis of expression of podoplanin and VEGF-C in podoplanin-overexpressing cells. Actin expression was used as an internal control.

podoplanin-expressing FaDu and SNU-1041 were increased to 148.3 and $113.9 \%$ of that in the mock vector-expressing FaDu and SNU-1041 cells, respectively (Fig. 4B). Furthermore, overexpression of podoplanin led to an increase in the VEGF-C transcript level (Fig. 4C) and protein expression (Fig. 4D) in the FaDu and SNU-1041 cells, respectively. Other VEGF family such as VEGF-A, -B and -D were not altered in the podoplanin-overexpressing cells compared to that in the mock cells (Fig. 4C). These results suggest that podoplanin regulates cell wound healing activity and invasiveness through interaction of VEGF-C in HNSCC cells.

Podoplanin regulates the metastatic process through interaction of VEGF-C in the HNSCC cells. To determine whether podoplanin modulates HNSCC cell metastasis, we examined cell wound healing migration and a Transwell invasion assay in VEGF-C-suppressed cells transfected with VEGF-C siRNA. We confirmed that VEGF-C protein expression was suppressed in the VEGF-C siRNA-expressing FaDu and SNU-1041 cells compared with levels in the control siRNA-expressing FaDu and SNU-1041 cells, respectively (Fig. 5A). Control siRNA-expressing FaDu and SNU-1041 cells repaired the wound area rapidly at $24 \mathrm{~h}$, while the VEGF-C siRNA-expressing FaDu and SNU-1041 cells suppressed the wound repair significantly at $24 \mathrm{~h}$, respectively (Fig. 5A). In addition, the percentage of invasive cells in the VEGF-C siRNA-expressing FaDu and SNU-1041 were decreased to 71.9 and $75.4 \%$ of that in the control siRNA-expressing FaDu and SNU-1041 cells, respectively (Fig. 5B). Additionally, inhibition of VEGF-C led to suppression of podoplanin expression in the FaDu (Fig. 5C) and SNU-1041 (Fig. 5D) cells, respectively.

Next, to determine a correlation between podoplanin and VEGF-C, we examined the cell wound healing migration and invasion assays in both podoplanin overexpression vector and VEGF-C siRNA-cotransfected FaDu and SNU-1041 cells, respectively. The podoplanin-overexpressing $\mathrm{FaDu}$ and SNU-1041 cells rapidly repaired the wound area compared with this rate in the mock cells, respectively (Fig. 6A and C). In contrast, both podoplanin vector and VEGF-C siRNAcoexpressing FaDu or SNU-1041 cells inhibited wound repair compared with the podoplanin vector-expressing $\mathrm{FaDu}$ and SNU-1041 cells (Fig. 6A and C). In addition, the percentages of invasive cells in the podoplanin vector-expressing FaDu and SNU-1041 were increased to 163.9 and $184 \%$ of that in the mock vector-expressing FaDu and SNU-1041 cells (Fig. 6B and D). In the cell wound healing assay, the percentages of invasive cells in both the podoplanin vector and VEGF-C siRNA-coexpressing FaDu and SNU-1041 cells were 
A

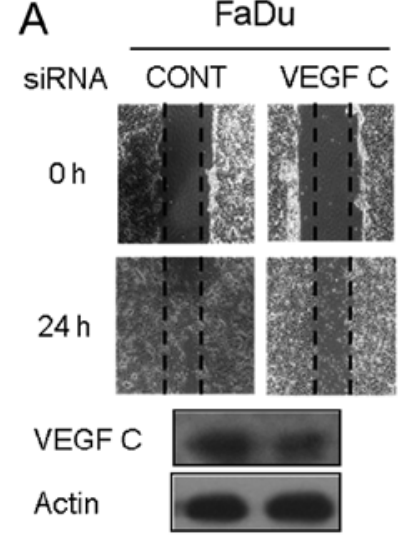

B 120

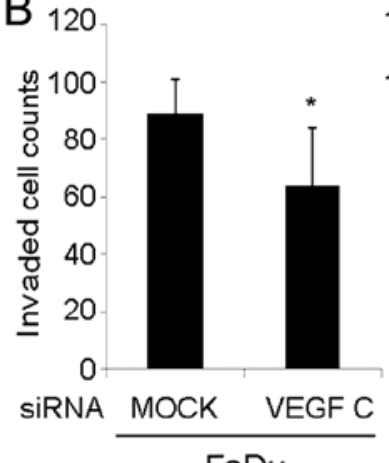

$\mathrm{FaDu}$
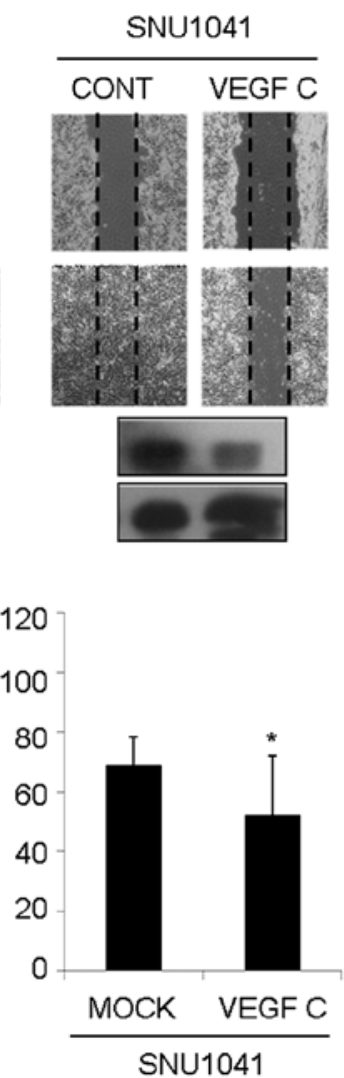

C FaDu

\begin{tabular}{|c|c|c|c|c|c|c|}
\hline+ & - & - & - & - & - & MOCK DNA \\
\hline- & + & + & + & - & - & PDPN DNA \\
\hline - & - & + & - & + & - & CONT siRNA \\
\hline- & - & - & + & - & + & VEGF C siRN \\
\hline
\end{tabular}

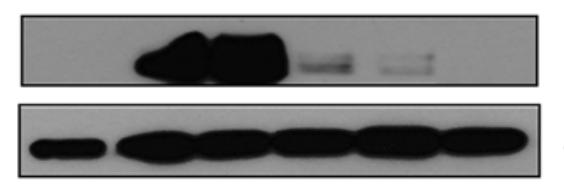

PDPN

Actin

D

SNU1041

\begin{tabular}{|c|c|c|c|c|c|c|}
\hline+ & - & - & - & - & - & MOCK DNA \\
\hline- & + & + & + & - & - & PDPN DNA \\
\hline- & - & + & - & + & - & CONT siRNA \\
\hline- & - & - & + & - & + & VEGF C siRN \\
\hline
\end{tabular}

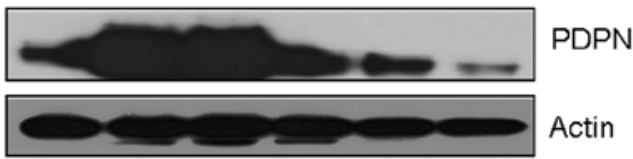

Figure 5. Suppression of cell wound healing migration and invasion in VEGF-C siRNA-expressing FaDu and SNU-1041 cells. (A) The wound healing assay was conducted by scraping a wound area. Cells were monitored using phase-contrast microscopy at $24 \mathrm{~h}$ after transfection of VEGF-C siRNA. (B) The invasion assay was carried out according to Patients and methods. The mean values and the standard error were obtained from three individual experiments. ${ }^{*} \mathrm{P}<0.1$. (C and D) Western blot analysis of expression of podoplanin was analyzed in both podoplanin vector and VEGF-C siRNA-coexpressing cells. Actin expression was used as an internal control.

significantly decreased to 48.8 and $20.7 \%$ of these percentage in the podoplanin vector-expressing FaDu and SNU-1041 cells, respectively (Fig. 6B and D). These results indicated that podoplanin regulates metastasis via interaction of VEGF-C in HNSCC cells.

\section{Discussion}

Podoplanin is a $38-\mathrm{kDa}$ type I transmembrane glycoprotein consisting of 162 amino acids that is expressed almost exclusively in lymphatic endothelial cells (19). In other normal human tissues, podoplanin is expressed by various cells, including kidney podocytes, lung alveolar type I cells, basal epithelial keratinocytes of the skin, cervix and esophagus and myoepithelial cells of the breast gland and salivary glands (20). Due to its specific expression in lymphatic endothelium, podoplanin has been widely used as a specific marker of lymphatic endothelial cells and lymphangiogenesis under physiological and pathological conditions. Thus, over the past few decades, podoplanin has been used to assess tumoral lymphatic vessel density in many types of cancer and correlates with lymph node metastasis and poor prognosis (14,21-23).

Podoplanin expression is upregulated in a number of human cancer types, including squamous cell carcinomas of the oral cavity, lung, cervix, esophagus and the skin, in dysgerminomas of the ovary and granulosa cell tumors, in mesothelioma and in many tumors of the central nervous system (9-15,24-27). Recent experimental results have also demonstrated that podoplanin mediates a pathway leading to collective cell migration and invasion in vivo and in vitro (27). Some investigators have demonstrated a possible relationship between podoplanin expression and tumor invasion or metastasis $(11,12,14,21)$. In oral leukoplakia, high podoplanin expression has been associated with an increased risk of progression to invasive cancer, suggesting that podoplanin may be a powerful biomarker of the risk of oral cancer development in patients with oral leukoplakia (15). This evidence supports the importance of podoplanin in oral tumorigenesis and malignant transformation. Recent studies have also shown that high levels of podoplanin expression in primary oral SCC are associated with advanced T stage, lymphatic spread to the cervical region and a poor clinical outcome $(14,28)$. Tong et al demonstrated a significant association between the level of podoplanin expression and depth of tumor invasion, lymph node status, lymphatic vessel density, progressive TNM stage and disease-free survival time in patients with esophageal SCC (29). Thus, they suggested that podoplanin may be useful as an independent prognostic factor for esophageal SCC. In the present study, we also found that podoplanin was strongly associated with lymph node metastasis and poor histological grade in HNSCC patients. More importantly, high levels of podoplanin expression were associated with decreased overall and disease-specific survival rate in patients with HNSCC. 
A

$\mathrm{FaDu}$

Oh
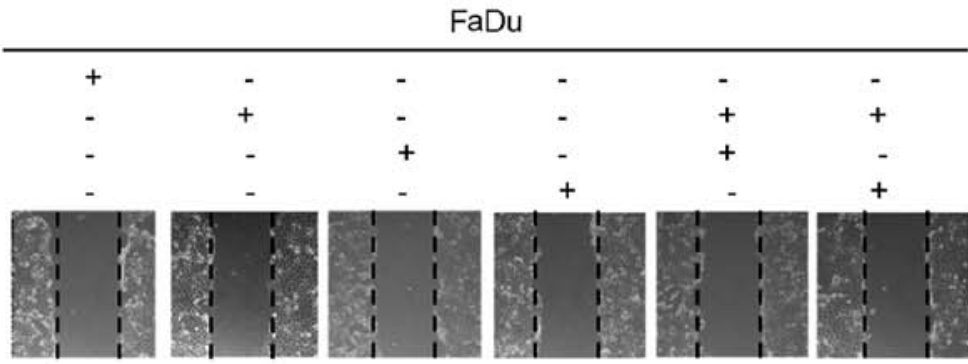

MOCK DNA

PDPN DNA

CONT SIRNA

$8 \mathrm{~h}$
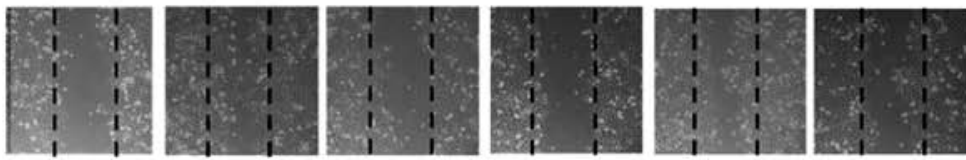

VEGF C SIRNA

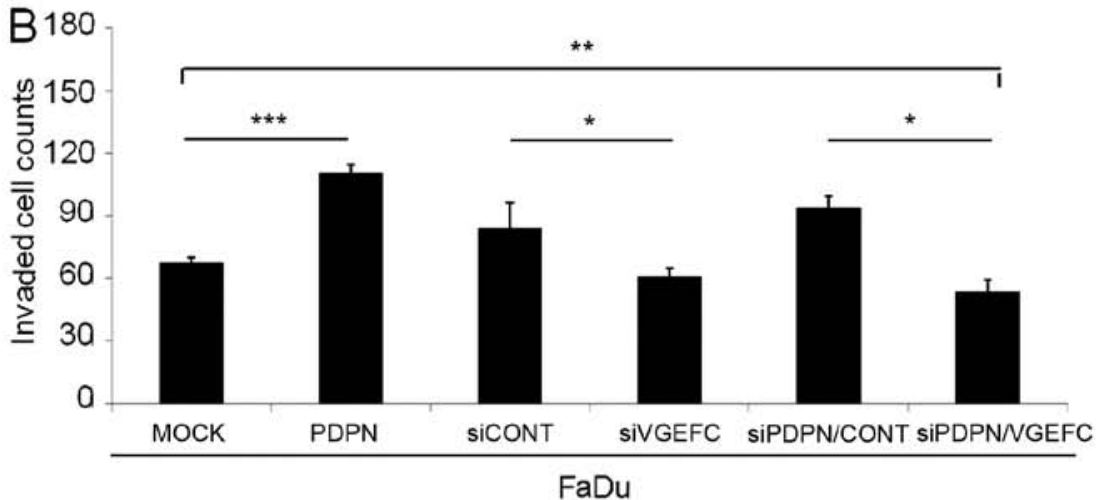

C

SNU1041
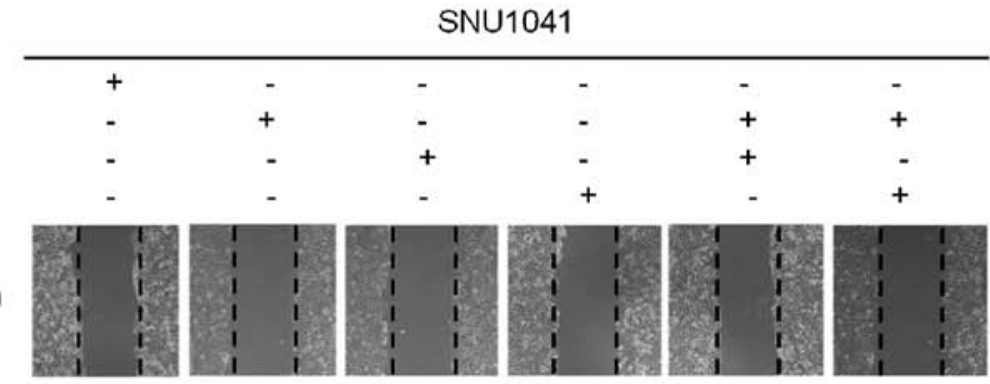

MOCK DNA

PDPN DNA

CONT SIRNA

$18 \mathrm{~h}$
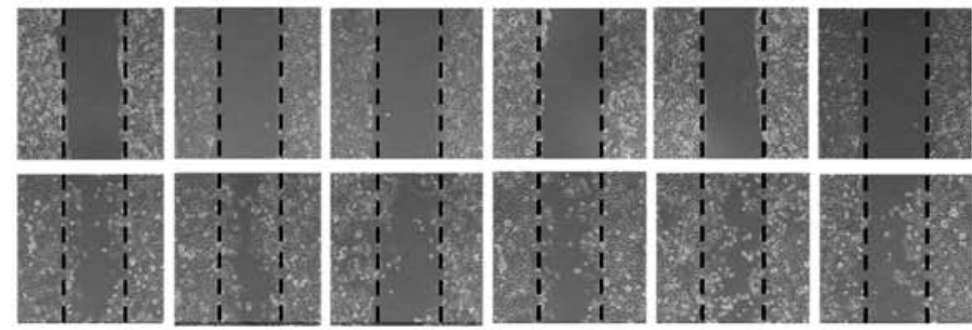

VEGF C SIRNA

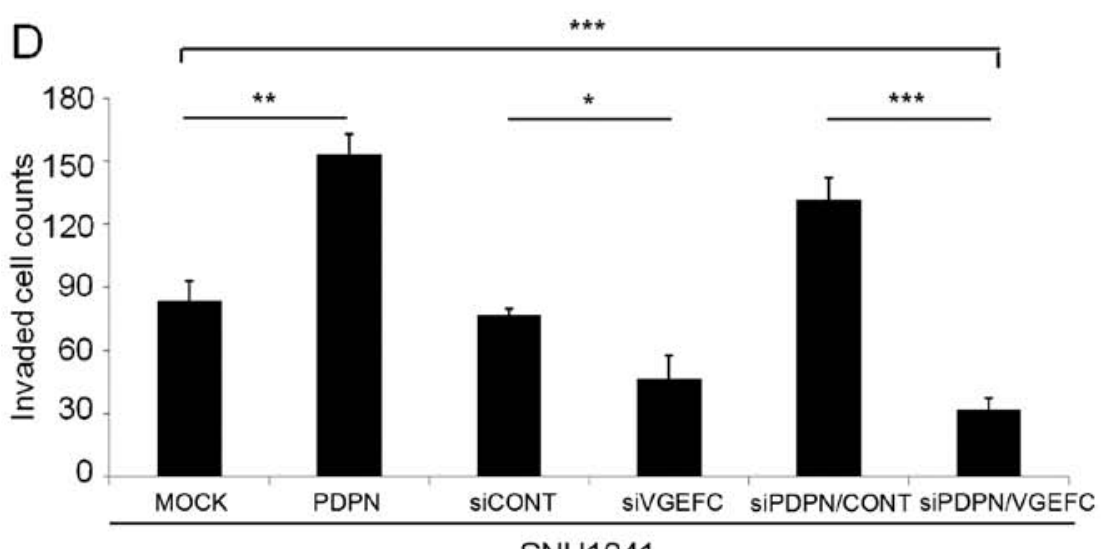

SNU1041

Figure 6. Suppression of cell wound healing migration and invasion in both podoplanin vector and VEGF-C siRNA-coexpressing FaDu and SNU-1041 cells. (A and C) The wound healing assay was conducted by scraping a wound area. Cells were monitored using phase-contrast microscopy at $24 \mathrm{~h}$ after cotransfection of both podoplanin vector and VEGF-C siRNA in FaDu and SNU-1041 cells, respectively. (B and D) The invasion assay was performed in both podoplanin vector and VEGF-C siRNA-cotransfected FaDu and SNU-1041 cells, respectively. The mean values and the standard error were obtained from three individual experiments. ${ }^{*} \mathrm{P}<0.05,{ }^{* * *} \mathrm{P}<0.005$ and ${ }^{* * * *} \mathrm{P}<0.005$. 
However, in contrast to these findings, Dumoff et al (11) reported a strong correlation between low expression of podoplanin and both lymphatic invasion and nodal metastasis in uterine cervical cancer. Rodrigo et al (30) reported that laryngeal cancer patients with high podoplanin expression showed prolonged disease-specific survival. These findings suggest that the biological function of podoplanin may vary according to cancer type. Considering that only 48 laryngeal carcinoma cases were investigated in the present study, high podoplanin expression was also significantly correlated with poor survival outcome, in contrast to the results of Rodrigo et al (30). This may have been since most of the laryngeal carcinoma patients had advanced-stage disease and underwent total laryngectomies, and the case number of laryngeal carcinoma patients was small in our study population. The prognostic effects of podoplanin expression should be investigated in larger populations of laryngeal carcinoma patients.

Our results suggest that podoplanin plays a role in lymphatic spread and tumor progression in HNSCC, while the exact mechanism is unclear. It has been demonstrated that podoplanin contributes to tumor invasion by binding ERM proteins, such as ezrin, radixin and moesin to activate RhoA, resulting in epithelial-mesenchymal transition (EMT) (31). However, Wicki et al (27) suggested that podoplanin induced collective tumor cell invasion in the absence of a cadherin switch or EMT. Podoplanin is also involved in the aggregation of platelets and may therefore enhance the arrest, extravasation and subsequent metastasis of podoplanin-expressing tumor cells circulating in the blood (32). In the present study, we found that silencing of podoplanin led to suppression of VEGF-C expression resulting in inhibition of cell wound healing repair and invasion (Fig. 3) and that overexpression of podoplanin led to elevation of VEGF-C expression resulting in induction of metastatic processes such as wound healing migration and invasion in HNSCC cells (Fig. 4). As shown in Fig. 5 and 6, treatment of VEGF-C siRNA and overexpression of podoplanin suppressed wound repair activity and invasiveness in both FaDu and SNU-1041 cells. Based on these results, we suggest that podoplanin regulates metastatic processes such as migration and invasion via interaction of VEGF-C in HNSCC cells. Research is ongoing to elucidate the detailed molecular mechanisms underlying the relationship between podoplanin and VEGF-C in the metastatic process of HNSCC.

In conclusion, our findings suggest that high podoplanin expression is associated with aggressive tumor behavior, poor prognosis of head and neck cancer and regulation of metastasis through VEGF-C modulation in HNSCC. Podoplanin may be a potential regulator of VEGF-C in HNSCC metastasis and may be used as a prognostic biomarker for HNSCC patients.

\section{Acknowledgements}

The present study was supported by the National Research Foundation of Korea (NRF) grant funded by the Korea government (MSIP) (nos. 2013R1A2A2A01015281 and 2012R1A1A2005393) and partially supported by a grant from the National R\&D Program for Cancer Control, Ministry of Health, Welfare and Family Affairs, Republic of Korea (0720560).

\section{References}

1. Parkin DM, Bray F, Ferlay J and Pisani P: Global cancer statistics, 2002. CA Cancer J Clin 55: 74-108, 2005.

2. Forastiere A, Koch W, Trotti A and Sidransky D: Head and neck cancer. N Engl J Med 345: 1890-1900, 2001.

3. Capote A, Escorial V, Muñoz-Guerra MF, Rodríguez-Campo FJ, Gamallo $\mathrm{C}$ and Naval L: Elective neck dissection in early-stage oral squamous cell carcinoma - does it influence recurrence and survival? Head Neck 29: 3-11, 2007.

4. Kowalski LP, Bagietto R, Lara JR, Santos RL, Silva JF Jr and Magrin J: Prognostic significance of the distribution of neck node metastasis from oral carcinoma. Head Neck 22: 207-214, 2000.

5. Lothaire P, de Azambuja E, Dequanter D, Lalami Y, Sotiriou C, Andry G, Castro G Jr and Awada A: Molecular markers of head and neck squamous cell carcinoma: Promising signs in need of prospective evaluation. Head Neck 28: 256-269, 2006.

6. Lopes MA, Nikitakis NG, Reynolds MA, Ord RA and Sauk J Jr: Biomarkers predictive of lymph node metastases in oral squamous cell carcinoma. J Oral Maxillofac Surg 60: 142-147, discussion 147-148, 2002.

7. Kahn HJ and Marks A: A new monoclonal antibody, D2-40, for detection of lymphatic invasion in primary tumors. Lab Invest 82 : 1255-1257, 2002.

8. Schacht V, Ramirez MI, Hong YK, Hirakawa S, Feng D, Harvey N, Williams M, Dvorak AM, Dvorak HF, Oliver G, et al: T1alpha/podoplanin deficiency disrupts normal lymphatic vasculature formation and causes lymphedema. EMBO J 22: 3546-3556, 2003.

9. Durchdewald M, Guinea-Viniegra J, Haag D, Riehl A, Lichter P, Hahn M, Wagner EF, Angel P and Hess J: Podoplanin is a novel fos target gene in skin carcinogenesis. Cancer Res 68: 6877-6883, 2008.

10. Shimada Y, Ishii G, Nagai K, Atsumi N, Fujii S, Yamada A, Yamane Y, Hishida T, Nishimura M, Yoshida J, et al: Expression of podoplanin, CD44, and p63 in squamous cell carcinoma of the lung. Cancer Sci 100: 2054-2059, 2009.

11. Dumoff KL, Chu CS, Harris EE, Holtz D, Xu X, Zhang PJ and Acs G: Low podoplanin expression in pretreatment biopsy material predicts poor prognosis in advanced-stage squamous cell carcinoma of the uterine cervix treated by primary radiation. Mod Pathol 19: 708-716, 2006.

12. Chuang WY, Yeh CJ, Wu YC, Chao YK, Liu YH, Tseng CK, Chang HK, Liu HP and Hsueh C: Tumor cell expression of podoplanin correlates with nodal metastasis in esophageal squamous cell carcinoma. Histol Histopathol 24: 1021-1027, 2009.

13. Rahadiani N, Ikeda J, Makino T, Tian T, Qiu Y, Mamat S, Wang Y, Doki Y, Aozasa K and Morii E: Tumorigenic role of podoplanin in esophageal squamous-cell carcinoma. Ann Surg Oncol 17: 1311-1323, 2010.

14. Yuan P, Temam S, El-Naggar A, Zhou X, Liu DD, Lee JJ and Mao L: Overexpression of podoplanin in oral cancer and its association with poor clinical outcome. Cancer 107: 563-569, 2006.

15. Kawaguchi H, El-Naggar AK, Papadimitrakopoulou V, Ren H, Fan YH, Feng L, Lee JJ, Kim E, Hong WK, Lippman SM, et al: Podoplanin: A novel marker for oral cancer risk in patients with oral premalignancy. J Clin Oncol 26: 354-360, 2008.

16. Byrd DR, Compton CC, Fritz AG, Greene FL, Trotti A (eds): AJCC Cancer Staging Manual. 7th edition. Springer, New York, NY, 2009.

17. Koo BS, Kim JM, Seo ST, Yoon YH, Kwon KR, Kim SH, Kwon HW, Bae WJ and Lim YC: Upregulation of HGF and c-MET is associated with subclinical central lymph node metastasis in papillary thyroid microcarcinoma. Ann Surg Oncol 21: 2310-2317, 2014

18. Kang YH, Ji NY, Han SR, Lee CI, Kim JW, Yeom YI, Kim YH, Chun HK, Kim JW, Chung JW, et al: ESM-1 regulates cell growth and metastatic process through activation of NF- $\mathrm{KB}$ in colorectal cancer. Cell Signal 24: 1940-1949, 2012.

19. Schoppmann SF, Birner P, Studer P and Breiteneder-Geleff S: Lymphatic microvessel density and lymphovascular invasion assessed by anti-podoplanin immunostaining in human breast cancer. Anticancer Res 21: 2351-2355, 2001.

20. Raica M, Cimpean AM and Ribatti D: The role of podoplanin in tumor progression and metastasis. Anticancer Res 28: 2997-3006, 2008. 
21. Tomita N, Matsumoto T, Hayashi T, Arakawa A, Sonoue H, Kajiyama Y and Tsurumaru M: Lymphatic invasion according to D2-40 immunostaining is a strong predictor of nodal metastasis in superficial squamous cell carcinoma of the esophagus: Algorithm for risk of nodal metastasis based on lymphatic invasion. Pathol Int 58: 282-287, 2008.

22. Schacht V, Dadras SS, Johnson LA, Jackson DG, Hong YK and Detmar M: Up-regulation of the lymphatic marker podoplanin, a mucin-type transmembrane glycoprotein, in human squamous cell carcinomas and germ cell tumors. Am J Pathol 166: 913-921, 2005.

23. Erovic BM, Neuchrist C, Kandutsch S, Woegerbauer M and Pammer J: CD9 expression on lymphatic vessels in head and neck mucosa. Mod Pathol 16: 1028-1034, 2003.

24. Kato Y, Kaneko M, Sata M, Fujita N, Tsuruo T and Osawa M: Enhanced expression of Aggrus (T1alpha/podoplanin), a plateletaggregation-inducing factor in lung squamous cell carcinoma. Tumour Biol 26: 195-200, 2005.

25. Kimura $\mathrm{N}$ and Kimura I: Podoplanin as a marker for mesothelioma. Pathol Int 55: 83-86, 2005.

26. Shibahara J, Kashima T, Kikuchi Y, Kunita A and Fukayama M Podoplanin is expressed in subsets of tumors of the central nervous system. Virchows Arch 448: 493-499, 2006.
27. Wicki A, Lehembre F, Wick N, Hantusch B, Kerjaschki D and Christofori G: Tumor invasion in the absence of epithelialmesenchymal transition: Podoplanin-mediated remodeling of the actin cytoskeleton. Cancer Cell 9: 261-272, 2006.

28. Kreppel M, Scheer M, Drebber U, Ritter L and Zöller JE: Impact of podoplanin expression in oral squamous cell carcinoma: Clinical and histopathologic correlations. Virchows Arch 456: 473-482, 2010.

29. Tong L, Yuan S, Feng F and Zhang H: Role of podoplanin expression in esophageal squamous cell carcinoma: A retrospective study. Dis Esophagus 25: 72-80, 2012.

30. Rodrigo JP, García-Carracedo D, González MV, Mancebo G, Fresno MF and García-Pedrero J: Podoplanin expression in the development and progression of laryngeal squamous cell carcinomas. Mol Cancer 9: 48, 2010.

31. Martín-Villar E, Megías D, Castel S, Yurrita MM, Vilaró S and Quintanilla M: Podoplanin binds ERM proteins to activate RhoA and promote epithelial-mesenchymal transition. J Cell Sci 119: 4541-4553, 2006.

32. Cueni LN, Hegyi I, Shin JW, Albinger-Hegyi A, Gruber S, Kunstfeld R, Moch H and Detmar M: Tumor lymphangiogenesis and metastasis to lymph nodes induced by cancer cell expression of podoplanin. Am J Pathol 177: 1004-1016, 2010. 